\title{
Effects of Single and Repeated Intravitreal Applications of Atropine on Choroidal Thickness in Alert Chickens
}

\author{
Ute Mathis $^{\mathrm{a}}$ Marita Pauline Feldkaemper ${ }^{\mathrm{a}}$ Frank Schaeffel ${ }^{\mathrm{a}, \mathrm{b}, \mathrm{c}}$ \\ aOphthalmic Research Institute, Section of Neurobiology of the Eye, University of Tuebingen, Tuebingen, Germany; \\ ${ }^{b}$ Institute for Molecular and Clinical Ophthalmology Basel (IOB), Basel, Switzerland; ' Zeiss Vision Lab, Ophthalmic \\ Research Institute, University of Tuebingen, Tuebingen, Germany
}

\section{Keywords}

Myopia $\cdot$ Atropine $\cdot$ Choroid $\cdot$ Chicken

\begin{abstract}
Introduction: Atropine, a muscarinic antagonist, is known since the 19th century to inhibit myopia development in children. One of its effects is that it stimulates choroidal thickening. Thicker choroids, in turn, have been linked to myopia inhibition. We used the atropine-stimulated choroidal response in the chicken to learn more about the time courses and amplitudes of the effects of atropine, as well as whether repeated applications lead to accumulation or desensitization. Methods: Intravitreal injections containing $250 \mu \mathrm{g}$ atropine sulfate were performed in 1 eye around 10:00 in the morning, the fellow eye received vehicle. Chickens with bilateral vehicle injections served as controls. Choroidal thickness was measured over the day for every 2-3 $\mathrm{h}$ in alert animals, using spectral domain optical coherence tomography, with 3-5 independent measurements in each eye. Three experiments were done - (1) single injection and time course measured over 1 day, (2) single injection and time course measured over 4 days, and (3) daily injections and time course measured over 4 days for measuring the ef-
\end{abstract}

fects of atropine on vitreal, retinal, and choroidal dopamine, and 3,4-dihydroxyphenylacetic acid levels by using highperformance liquid chromatography with electrochemical detection. Results: Atropine induced an increase in choroidal thickness by about 60 percent, with a peak amplitude after about $2 \mathrm{~h}$. The effect persisted only for a few hours and had nearly disappeared by evening. Initially, similar amounts of choroidal thickening were observed in vehicle-injected fellow eyes but recovery to baseline was faster. When atropine was injected daily for 4 days, choroids thickened every day with similar amplitudes and time courses, with no signs of either accumulation or desensitization effects. Interestingly, while dopamine release from the retina was stimulated by atropine and followed approximately, the time course of choroidal thickening, its tissue concentration dropped in the choroid. Conclusions: Even at relatively high intravitreal doses, effects of atropine on choroidal thickness remained transient, similar to its effects on retinal dopamine. With repeated application every day, the diurnal patterns of choroidal thickening could be reproduced for 4 days with similar amplitudes and time courses. The transient nature of the effects of atropine on the choroid may be relevant for application protocols of atropine against myopia.

(c) 2021 The Author(s).

Published by S. Karger AG, Basel karger@karger.com www.karger.com/ore

Karger $\stackrel{\text { ! }}{=}$

BOPEN ACCESS
(C) 2021 The Author(s)

Published by S. Karger AG, Basel

This is an Open Access article licensed under the Creative Common Attribution-NonCommercial-4.0 International License (CC BY-NC) (http://www.karger.com/Services/OpenAccessLicense), applicable to the online version of the article only. Usage and distribution for commercial purposes requires written permission.
Correspondence to:

Frank Schaeffel, frank.schaeffel@uni-tuebingen.de 


\section{Introduction}

Atropine is currently the most frequently used drug to inhibit myopia in children and was considered "an important step forward in myopia prevention" [1]. However, despite considerable research efforts, knowledge about its mechanisms to inhibit myopia remains incomplete [2]. Atropine has a number of effects that might be related to its inhibitory effect on myopia. In the chicken model of myopia, intravitreal injection of atropine stimulates retinal release of dopamine [3], which is assumed to be involved in the signaling cascade of visually controlled eye growth (for reviews, see $[4,5]$ ). Atropine also stimulates retinal expression of the transcription factor ZENK $[6,7]$ which was considered an eye growth-inhibiting signal [8]. Furthermore, atropine was found to thicken the choroid in young adult human subjects [9] and in children [10], where high concentrations $(0.5$ and $1 \%$ atropine gel, respectively) were used to show the effect. Later, it was found that low dose $(0.01 \%)$ atropine eye drops also induce the choroidal thickening in children [11]. Atropine also interacts with lens defocus-induced changes in choroidal thickness - it prevents choroidal thinning induced by short term exposure to negative lenses in young adults [12]. Most recently, a study in Taiwanese school children [13] showed that daily application of $0.3 \%$ atropine eye drops for 1 week caused the choroid to thicken significantly. With continued atropine application, the choroid remained thick over an observation period of 6 months. No choroidal thinning could be induced during this period when children wore a $-2 \mathrm{D}$ lens for $1 \mathrm{~h}$, while $\mathrm{a}+2 \mathrm{D}$ lens continued to cause further thickening of the choroid. That atropine which can increase the choroidal thickness is of interest since it was linked to inhibition of myopia development in previous studies, as discussed below.

\section{Predictive Value of Choroidal Thickness for Future Refractive Development: Evidence from Animal Models and Children}

Wallman and colleagues [14] were the first to describe that the choroid can double its thickness in 2 days in young chickens when they recover from experimentally induced myopia. It was calculated that the thickening choroid can temporarily correct for up to 7D of myopia by moving the retina closer to the focal plane. In humans, the effects on refraction would be much smaller. A schematic human eye [15] becomes about 3D my- opic if it elongates by $1 \mathrm{~mm}$. The changes in choroidal thickness that are elicited by $0.3 \%$ atropine eye drops were $21 \mu \mathrm{m}$ [13] which would be equivalent to only $0.063 \mathrm{D}$. Wearing positive lenses also induced choroidal thickening and reduced the myopic refractive error imposed by the lenses. Nickla and Totonelly [16] found that "choroidal thickness predicted ocular growth rates in normal eyes: eyes with thinner choroids grew faster than those with thicker choroids." However, they also found that this correlation was disrupted when refractive errors were experimentally induced by lenses. A similar conclusion was already drawn in a previous publication by Nickla et al. [17] when it was found that choroidal and scleral diurnal rhythms can be phase-shifted with respect to each other when refractive errors were induced by lenses or diffusers. Accordingly, they proposed that the mechanisms for the control of choroidal thickness and scleral growth may be distinct. Nickla et al. [18] found that inhibition of the transient choroidal thickening response by the nitric oxide synthase inhibitor L-NAME also prevented inhibition of the axial eye growth by positive lenses, further supporting the idea of a link between an increase in choroidal thickness and inhibition of the axial eye growth. Jiang et al. [19] studied 2 strains of guinea pigs, one of them developing very little myopia with diffusers, the other becoming much more myopic. Interestingly, the strain developing little myopia also had much thicker choroids from the beginning which led the authors speculate that a thicker choroid might be protective against the development of deprivation myopia. Visually induced choroidal thickness changes were observed in marmosets [20] and rhesus monkeys also [21].

Like in animal models, changes in choroidal thickness could be experimentally induced in children also. Wang et al. [22] found that $2 \mathrm{~h}$ of monocular exposure to positive lenses (or myopic defocus) induced choroidal thickening, while negative lenses induced choroidal thinning in 8-16 year old, largely myopic school children. Positive or negative defocus was imposed by adding $+3 \mathrm{D}$ or $-3 \mathrm{D}$ lenses, respectively, to their habitual corrections. The authors propose that "this rapid and reversible choroidal response may be an important clinical parameter in gauging retinal responses to optical defocus in human myopia." Studies on the development of myopia in children also support a role of choroidal thickness changes in refractive development. School children (10-15 years old) who displayed less choroidal thickening with age also had faster eye growth and became more myopic [23]. Similarly, Jin et al. [24] observed that choroidal thinning oc- 
curs in 7-12-year-old children early in myopic progression although no correlation was found to axial length. That choroidal thickness which may serve as a marker for myopic progression in children was also concluded by Prousali et al. [25] and Fontaine et al. [26] who proposed that "a thinner choroid may predict the onset, or progression, of myopia."

\section{Rationale for the Current Study}

Considering the evidence that choroidal thickening is linked to inhibition of myopia, and that atropine can thicken the choroid, it is important to determine effect sizes and time courses of atropine-induced choroidal thickening. Furthermore, since atropine is known to stimulate the release of dopamine from the retina, it is of interest to compare the kinetics of choroidal thickness changes and retinal dopamine release. It is also not known whether repeated intravitreal atropine applications can increase treatment effects due to a possible accumulation of atropine, or whether they can result in desensitization of the (unknown) receptor mechanisms. Moreover, it is important to know whether unilateral application of atropine can also affect the choroid in the fellow eye. We have studied these questions in the chicken model.

\section{Material and Methods}

\section{Animals}

One-day-old male White Leghorn chickens were obtained from a local hatchery (Kilchberg, Germany). They were raised in temperature-controlled animal facilities under an 11/13-h light/ dark cycle (8:00 a.m.-7:00 p.m. or 8-19 h) at an illuminance of approximately 500 lux during the light phase. Water and food were supplied ad libitum. Animals were studied between post-hatch ages P14-P19. In total, 39 chickens were studied. All experiments were conducted in accordance with the ARVO statement for the use of animals in ophthalmic and vision research and were approved by the University Committee of Tuebingen for experiments involving animals (AK 01/19G).

\section{Intravitreal Injections}

Intravitreal injections of atropine sulfate monohydrate (250 $\mu \mathrm{g}$ in $25 \mu \mathrm{L}$ saline, $>97 \%$, Sigma Aldrich, Deisenhofen, Germany) were performed using a 0.5 - $\mathrm{mL}$ insulin syringe (30-gauge needle, BD Medical, Le Pont deClaix, France), while the fellow eye received $25 \mu \mathrm{L}$ saline. Alternatively, both eyes received $25 \mu \mathrm{L}$ saline. In previous experiments [27], it was found that daily injections of the selected atropine dose completely inhibited myopia induced by wearing -7D lenses. All injections were performed under mild ether anesthesia. The intravitreal injection procedure in the chicken is well established $[28,29]$ and, as in former and more recent studies (e.g., $[3,30,31])$, we never observed ocular inflammation.

\section{Treatments}

Experiment 1. Four chickens were randomly selected from a group of 8 to receive a single unilateral intravitreal atropine injection around 10:00 a.m., while the fellow eyes received a vehicle injection (experimental group). The other 4 animals received only vehicle injections in both eyes and served as controls to reveal possible effects of the injection itself. Optical coherence tomography (OCT) measurements were performed $1 \mathrm{~h}$ before, and about 1,3 , 4,6 , and $7 \mathrm{~h}$ after injections.

Experiment 2. Another group of 8 chickens was treated as in experiment 1 but choroidal thickness was measured $1 \mathrm{~h}$ before and 2, 3, 5, and $7 \mathrm{~h}$ after the injections around 10:00 a.m., and beyond for the following 3 days, with several measurements performed on each day.

Experiment 3. Further 9 chickens were split up into 4 binocularly vehicle-injected controls and 5 animals which received atropine injections in 1 eye and vehicle in the other. In this experiment, injections were repeated daily for the following 4 days. On each day, OCT measurements were performed $1 \mathrm{~h}$ before and 2, 3, 5, and $7 \mathrm{~h}$ after injection around 10:00 a.m. A final measurement was done on day 5 around 9:00 a.m.

Experiment 4. In 14 chickens (7 control and 7 experimental animals), atropine was injected as described above. They were euthanized after $1.5 \mathrm{~h}$ around 11:30 a.m. and their eyes enucleated and prepared for high-performance liquid chromatography (HPLC) with electrochemical detection to determine retinal, vitreous, and choroidal levels of dopamine and dihydroxyphenylacetic acid (DOPAC), one of its metabolites. At this time point, dopamine release was highest, as found in previous experiments [3].

\section{Measurements of Choroidal Thickness in Alert Chickens}

We had found earlier [31-34] that OCT represents a fast and convenient technique to measure choroidal thickness in alert chickens. Chicks were held by hand in front of an OCT (HRA + OCT Spectralis, SN 10543, 04/2016, Heidelberg Engineering; resolution mode: high speed, scan angle: $30^{\circ}$, scan type: B-scan, $768 \times 496$ pixels, line scan, eye tracking not engaged, scan rate: of the live image 8.8 frames/s, and measurements at 1,060 nm). For the measurements, the position of the chicken's head was manually aligned until the cornea was aligned about perpendicular to the axis of the OCT camera and the scan of the fundal layers became visible on the screen. Optimal alignment of the eye was assumed when the light patch representing the pupil in the left window on the screen was centered and a high contrast and horizontally aligned scan of the fundal layers became visible in the window on the right (e.g., see Fig. 1; a screenshot can be found in [31]). Misalignment of eyes during image capture resulted in tilting of scans, which was used as an indicator of the same and a need for realignment. Choroidal thickness was manually measured as the distance from the retinal pigment epithelium to the outer boundary of the choroid [34], using the publicly available software ImageJ (https://imagej.nih.gov/ij/). While the quality of the OCT scans of the fundal layers in alert chickens was not comparable to the quality of the scans in human fundal OCT imaging, the measurement procedure had undergone considerable testing and verification in the past in our laboratory. An interobserver correlation analysis for the OCT measurements of choroidal thickness was performed in a previous study in which both observers were blinded with regard to the treatment of the chickens [34] and showed a correlation coefficient of $R=0.937$. In the current study, standard deviations from repeated measurements in the same eyes ranged from 4 to $10 \mu \mathrm{m}$, considerably 


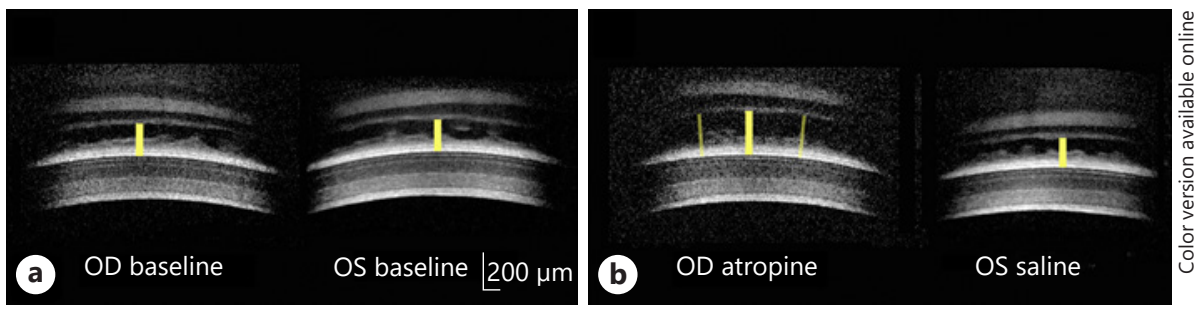

Fig. 1. Examples of OCT scans of the fundal layers in alert chickens. a Baseline thickness of the choroid in both eyes (about $200 \mu \mathrm{m}$, as denoted by the yellow bars). $\mathbf{b}$ The choroid in the atropine-injected eye is still considerably expanded after $3 \mathrm{~h}$, while the choroid in the fellow eye has already returned to baseline. In the scan of the atropine-treated eye, it is illustrated how 3 measurements of choroidal thickness were obtained in a single scan. They were subsequently averaged. OD, right eyes; OS, left eyes; OCT, optical coherence tomography.

Table 1. Main and interaction effects of atropine injections on choroidal thickness as determined by repeated measures ANOVA tween 20 and $50 \mu \mathrm{m})$. Five-ten OCT scans were stored for each eye and time point but only 3-5 were selected for analysis. Choroidal thickness was measured at 3 positions in the scan, 1 central and 2 positions left and right. Results were averaged. Scans were analyzed by 1 author who was blinded with regard to the treatment of the eyes.

\section{Measurements of Dopamine and Metabolites}

Animals were euthanized and the eyes were immediately enucleated. The eyeballs were cut with a razor blade into halves in the equatorial plane, approximately $1 \mathrm{~mm}$ posterior to the ora serrata. The anterior segment was discarded. The vitreous was removed and quickly frozen in liquid nitrogen. One $8-\mathrm{mm}$ tissue sample was taken from the posterior eye cup using a biopsy punch. The retina and choroid were isolated under a dissecting microscope, while the RPE cells were discarded. Tissues were frozen in liquid nitrogen and stored at $-80^{\circ} \mathrm{C}$ for subsequent HPLC analysis. All vitreous samples were weighed and homogenized in $750 \mu \mathrm{L}$ mobile phase (Thermo Fisher Scientific, Carlsbad, CA, USA) using a tissue lyser and 5-mm stainless steel beads (TissueLyser LT, Qiagen, Hilden, Germany) at $50 \mathrm{~Hz}$ for $4 \mathrm{~min}$. For retinal and choroidal samples, $500 \mu \mathrm{L}$ mobile phase was added before the homogenization. All homogenized samples were centrifuged at $4^{\circ} \mathrm{C}$ for $10 \mathrm{~min}$ at 14,000 $g$. The supernatant was filtered through a $0.2-\mu \mathrm{m}$ nylon membrane filter (Thermo Fischer Scientific, Rockwood, MI, USA), and $25 \mu \mathrm{L}$ was directly injected into the HPLC system. Samples were analyzed for catecholamine content by HPLC (Ultimate 3000 LC with electrochemical detection ECD3000RS, Thermo Fischer Scientific) with oculometric detection as previously described [3]. In brief, a hypersil C18 column was used $(150 \times 3 \mathrm{~mm}, 3 \mu \mathrm{m})$ together with a test mobile phase (Thermo Fischer Scientific) containing $10 \%$ acetonitrile and $1 \%$ phosphate buffer. The flow rate was $0.4 \mathrm{~mL} /$ min and the potential at the first and second electrode was set to +370 and $-200 \mathrm{mV}$, respectively. Dopamine and 3,4-DOPAC concentrations were determined with a high reproducibility (98\%). In the retina, biogenic amine content was determined as nanogram per milligram protein ( $\mathrm{ng} / \mathrm{mg}$ protein), whereas in vitreous and choroid, the amount of the substances was determined relative to wet weight (ng/0.1 g wet weight).

\section{Statistics}

Statistical analysis was done using commercial software "JMP 13" (SAS Institute, Cary, NC, USA). Data are shown as the mean

\begin{tabular}{|c|c|c|c|c|}
\hline & \multicolumn{2}{|c|}{ Experiment 1} & \multicolumn{2}{|c|}{ Experiment 2} \\
\hline & $F$ & $p$ value & $F$ & $p$ value \\
\hline Group & 6.342 & $0.012 *$ & 4.873 & $0.006^{* *}$ \\
\hline Time & 10.199 & $0.007 * *$ & 1.771 & 0.211 \\
\hline Group $\times$ time & 10.163 & $0.002^{* *}$ & 4.568 & $0.032^{*}$ \\
\hline
\end{tabular}

Significance levels $* p<0.05,{ }^{* *} p<0.01$.

\pm standard deviation or \pm standard errors as indicated, respectively. A mixed model repeated measure ANOVA (with time as the repeated measures and group as between subject factor) was used to compare mean choroidal thickness values between the different to compare the choroidal thickness in atropine-injected or contralateral, saline-injected eyes with that of saline-injected control animals at the same time point, respectively. Two-tailed paired Student's $t$-tests were used to compare atropine-injected and contralateral saline-injected eyes at the same time point. Bonferroni corrections were applied in the $t$-test post hoc analyses. Adjusted $p$ values of $p<0.05$ were considered to be significant. Also, for the results of HPLC measurements for the comparison of 2 independent groups, a two-tailed unpaired $t$-test was used. Differences in saline- and atropine-treated eyes in the same animal were compared using Student's 2-tailed paired $t$-tests.

\section{Results}

\section{Experiment 1: Effects of a Single Atropine Injection on} Choroidal Thickness over One Day

An example for the appearance of the fundal layers in the OCT scans is shown in Figure 1. Note that optical path lengths are measured by OCT, and not the geometrical, treatment groups. Two-tailed unpaired Student $t$-tests were used 
Fig. 2. a Experiment 1: after unilateral injection of atropine between 9 and 10:00 a.m., the choroid in both eyes displayed massive thickening which peaked after about $2 \mathrm{~h}$. After $5 \mathrm{~h}$, the width of the choroid in the saline-injected fellow eyes had returned to baseline, while it remained thick in the atropine-injected eye until the evening. ${ }^{*} p<0.05,{ }^{* * *} p<0.001$, the unpaired $t$-test of atropine-injected eyes versus eyes of vehicle-injected animals, ${ }^{\mathrm{xx}} p<$ 0.01 , the paired $t$-test of atropine-injected eyes versus contralateral control eyes. $\mathbf{b}$ Experiment 2: in a second group of chickens, choroidal thickness was followed for another 3 days. The time courses of choroidal thickness over the first day were perfectly reproduced from the first experiment. The choroid of atropine-injected eyes returned to baseline overnight, and there were no significant differences in choroidal thickness between the treatment groups over the following 3 days. Asterisks denote significance levels. ${ }^{* * *} p<0.001$, atropine-injected eyes versus vehicle-injected animals, ${ }^{\mathrm{xxx}} p<0.001$ atropine-injected eyes versus contralateral control eyes, ${ }^{\text {ooo }} p<0.001$ contralateral vehicle-injected eyes versus eyes of vehicle-injected animals. Note that the first data point in the graph refers to a baseline measurement before the injection.

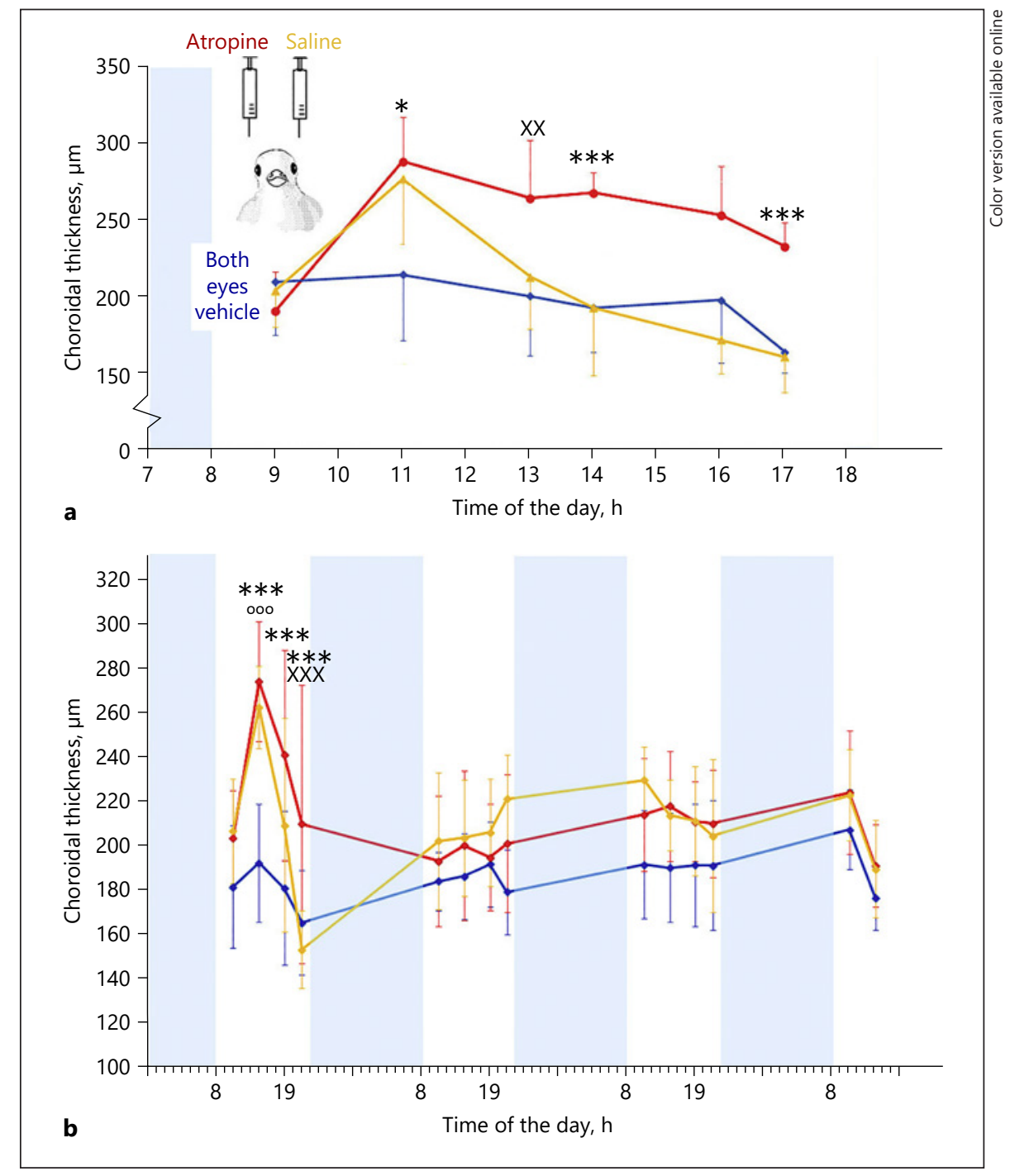

but comparisons of choroidal thickness before and after treatment are still valid. As determined by repeated measures ANOVA, the main effects of treatment groups (atropine-injected eyes, contralateral saline-injected eyes, and saline-injected controls) and time on choroidal thickness were significant. Additionally, the interaction effect of treatment and time on choroidal thickness was also significant (Table 1). One hour after unilateral atropine injection, the choroids of both eyes had thickened considerably, compared to baseline measurements before injections (delta choroidal thickness, mean \pm STD: saline-injected eyes: $71.9 \pm 37.3 \mu \mathrm{m}$; atropine-injected eyes: $96.5 \pm$ $42.5 \mu \mathrm{m})$. No changes in choroidal thickness were observed in bilaterally saline-injected controls. Another $2 \mathrm{~h}$ later, the choroid in the atropine-injected eyes was still thick, whereas the choroid in the fellow eyes had almost returned to baseline. Figure 2a shows that in the evening of the first day, atropine-injected eyes still had much thicker choroids (red lines), while their fellow eyes (orange lines) did no longer differ from control animals which had only received saline (blue lines).

\section{Experiment 2: Effects of a Single Atropine Injection on} Choroidal Thickness over Four Days

Because choroidal thickness in the atropine-treated eyes did not return to baseline at the end of the day, we added a second experiment and monitored choroidal thickness in atropine-treated and control animals over several days. As determined by repeated measures ANO$\mathrm{VA}$, the main effect of treatment groups (atropine-inject- 
Fig. 3. Experiment 3: long-term development of choroidal thickness when atropine was injected every morning for 4 days. Following atropine injection, choroidal thickness in atropine-treated eyes increased by about 60 percent every day (red line). In vehicle-injected fellow eyes, the increase was more variable but always significant, and showed faster recovery to baseline (yellow line). In binocularly vehicle-injected chickens, no clear trend was observed in choroidal thickness (blue line). These data show that a clear repetitive pattern is generated by daily injections.

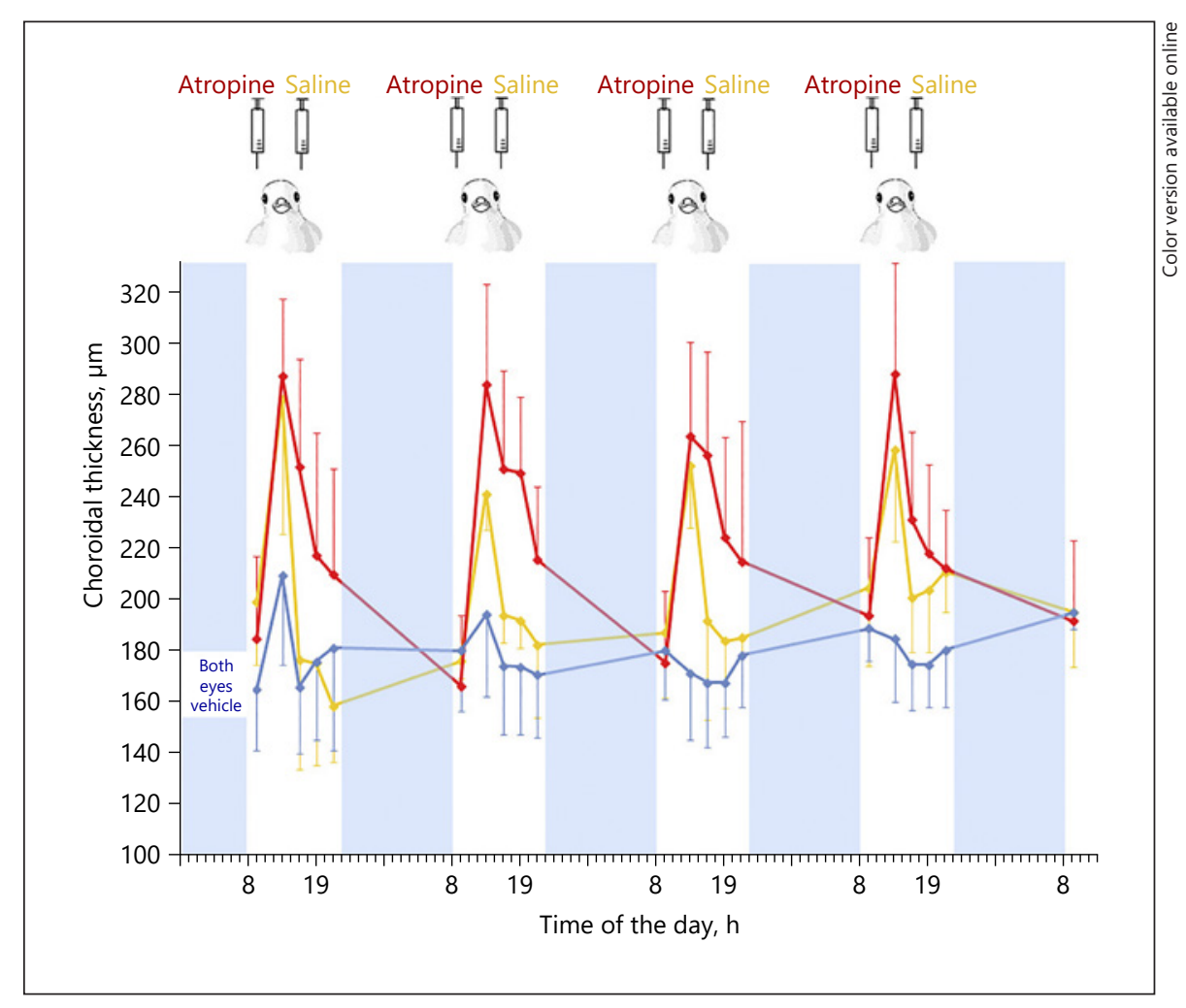

ed eyes, contralateral saline-injected eyes, and the salineinjected control group) again was significant, while time was not. Additionally, the interaction effect of group and time on choroidal thickness was significant (Table 1). It can be seen (Fig. 2b) that the time courses of choroidal thickness over the first day were perfectly reproduced from the first experiment. Second, it is evident that choroidal thickness in atropine-treated eyes had returned to baseline overnight. Over the following 3 days, there were no significant differences between atropine-treated, saline-treated fellow eyes, and eyes of only saline-injected control chickens.

\section{Experiment 3: Effects of Daily Atropine Injections on Choroidal Thickness over 4 days}

To determine whether atropine may lose some of its effect on the choroid with repeated applications, or whether the opposite is true due to atropine accumulation in tissues, a further experiment was done in which atropine was injected every morning for 4 days (Fig. 3). It can be seen that atropine injections increased choroidal thickness with a very similar time course and amplitude every day, with no signs of desensitization or accumulation. There were no significant atropine-induced changes in choroidal thickness over the 4-day treatment period - its effects remained strictly transient. Linear regressions of choroidal thickness over the 4-day treatment period were not significant $\left(R^{2}<0.01\right.$ in all 3 cases).

\section{Experiment 4: Changes in Vitreal, Retinal, and Choroidal Catecholamines after a Single Atropine Injection}

Since we had previously found [3] that intravitreal atropine injections stimulate dopamine release from the retina, we also studied changes in dopamine and its metabolite DOPAC in retina, vitreous, and choroid. Dopamine and DOPAC levels are shown in Figure 4. Dopamine levels were largely mirrored by the changes in DOPAC levels. While such a correlation cannot necessarily be expected since DOPAC levels measure dopamine turnover but dopamine levels do not, significant correlations were nevertheless observed in data of 25 eyes, except for the vitreous $(n=25$, retina: dopamine $[\mathrm{ng} / \mathrm{mg}$ protein $]=3.10 \times$ DOPAC $[\mathrm{ng} / \mathrm{mg}$ protein $]+1.64, R=$ $0.754, p<0.001$; vitreous: dopamine [ng/mg wet weight] $=0.05 \times \mathrm{DOPAC}[\mathrm{ng} / \mathrm{mg}$ wet weight $]+0.12, R=0.366$, $p<0.06$; and choroid: dopamine $[\mathrm{ng} / \mathrm{mg}$ wet weight $=$ $0.58 \times$ DOPAC $[\mathrm{ng} /$ wet weight $]+0.97, R=0.45, p<0.05)$. Dopamine levels in the vitreous and retina were increased 


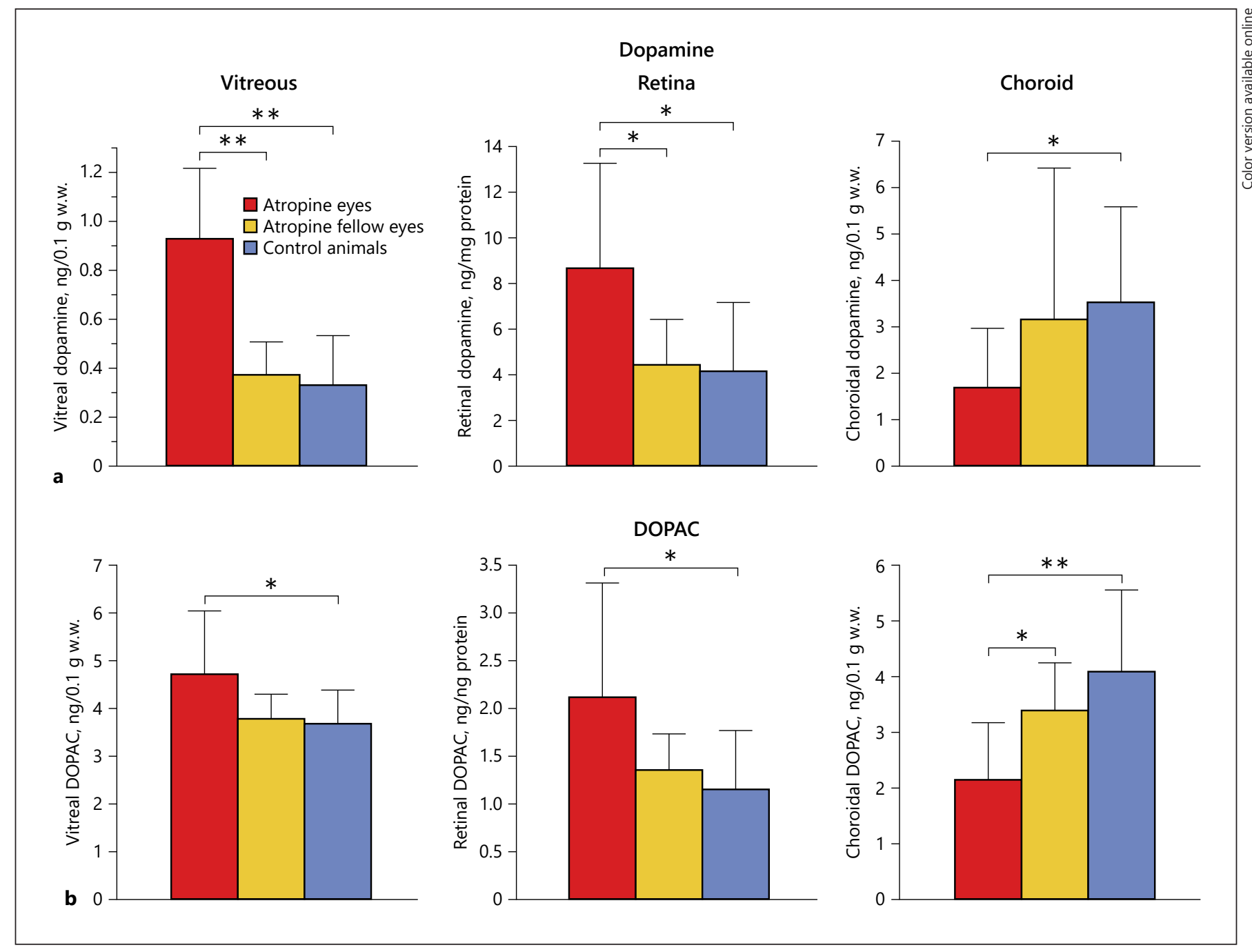

Fig. 4. Effects of a single atropine injection on vitreal, retinal, and choroidal dopamine (a) and DOPAC content (b). Atropine (red bar) increased dopamine content in the vitreous and retina relative to fellow eyes (yellow bar) and only in vehicle-injected chicks (blue bar), but lowered it in the choroid. Note that vitreal and choroidal dopamine and DOPAC contents were normalized to wet weight but retinal dopamine and DOPAC contents were normalized to protein content. ${ }^{* *} p<0.01 ;^{*} p<0.05$. DOPAC, dihydroxyphenylacetic acid.

\section{Discussion}

We found that a relatively high dose $(250 \mu \mathrm{g})$ of intravitreal atropine sulfate in chickens induces thickening of the choroid by about 60 percent. However, choroidal thickness returned to normal within 1 day. This result suggests that either atropine is rapidly removed from the tissue or that mechanisms triggered by atropine to thicken the choroid undergo rapid adaptation. We also found that repeated injections, every day in the morning over 4 days, induced highly reproducible changes in choroidal thickness with very similar amplitudes and time courses. 
There were no signs of desensitization or accumulation of atropine. Finally, we found that vehicle-injected fellow eyes showed similar changes in choroidal thickness, although with shorter duration and faster recovery to baseline. We can exclude, however, that these changes are just the result of the injections per se because animals which were only vehicle-injected did not display significant changes in choroidal thickness. Therefore, the effect in fellow eyes must also result from atropine. Contralateral effects could be explained either due to the distribution of atropine to the fellow eye via the blood stream, with systemic dilution [27] or by diffusion of atropine through the fundal layers into the other eye. In mice, it was found that the effects of a single $1 \%$ atropine eye drop on pupil size were very powerful and long-lasting ( 2 weeks) but only weak (25\%) in the untreated fellow eyes [35]. However, in mice both globes are more distant from each other while, in the chicken, the posterior poles of the 2 eyes are in close proximity, separated by only a thin cartilaginous plate [36].

\section{Dose Dependency of Choroidal Effects in the Chicken}

Even though we exposed the retina and choroid to atropine doses that were high enough to suppress myopia development completely in previous studies $(250 \mu \mathrm{g}$ atropine sulfate, equivalent to $0.24 \mathrm{~mm}$ in the vitreous [27]), the effects on choroidal thickness lasted only a limited period of time - no longer than 1 day. We did not establish a dose response function of choroidal thickness changes by injecting different doses of atropine but an indirect comparison of 2 doses is possible - comparing atropine-injected eye with saline-injected fellow eyes. An earlier study [27] concluded that fellow eyes were exposed to only about $1 / 8$ of the atropine concentration, compared to the atropine-injected eyes. The current data show that the initial amplitudes of choroidal thickening were only slightly lower in fellow eyes than in atropinetreated eyes. However, recovery of choroidal thickness to baseline was much faster in fellow eyes, indicating that the duration of choroidal effects of atropine increased with dose.

\section{Magnitudes of Atropine Effects on Choroidal}

\section{Thickness in Chickens and Humans}

In general, choroidal thickness changes are much larger in chickens than in humans. Wallman et al. [14] found that the choroid can thicken at least 2-fold during recovery from deprivation myopia. In the current study, atropine caused choroidal thickening in chickens by about $60 \%$. In Chinese children, $1 \%$ atropine gel topically ap- plied twice daily thickened the subfoveal choroid from $287.03 \pm 65.76 \mu \mathrm{m}$ to $302.52 \pm 69.94 \mu \mathrm{m}$ (equivalent to about 5\%) [10]. In a recent study by Chiang et al. [13] in Taiwanese school children, the choroid thickened from about 275 to $295 \mu \mathrm{m}$ (equivalent to about $7 \%$ ) when $0.3 \%$ atropine eye drops were applied daily for 1 week. Optically, these changes have almost no effect on refraction.

\section{Possible Mechanisms Underlying an Increase of Choroidal Thickness}

Wallman et al. [14] initially proposed that increased flow of aqueous into the uveoscleral path, and osmotically driven water movement into the choroid, triggered by increased choroidal proteoglycan synthesis, can thicken the choroid. They also proposed that the tonus of nonvascular smooth muscles which are distributed across the chicken choroid can antagonize these effects [37]. Blocking the action of acetylcholine on nonvascular smooth muscles by atropine could expand the choroid because these muscles relax. Choroidal expansion could also be mediated by an increase in choroidal blood flow. Using transcleral laser Doppler flowmetry, Fitzgerald et al. [38] found that choroidal blood flow was reduced during deprivation myopia but increased after $7 \mathrm{~h}$ of recovery. Interestingly, choroidal thickening occurred more slowly and continued after the blood flow was almost back to normal. The authors concluded that the increase in choroidal blood flow "may trigger or even drive the subsequent onset of choroidal expansion." Furthermore, leakage from choroidal blood vessels into the suprachoroidal fluid was studied as a potential mechanism for choroidal thickening by Rada and Palmer [39] and by Pendrak et al. [40], using albumin or fluorescein dextran as tracers. Fenestration of the walls of the lymphatics of the chick choroid were also studied by transmission electron microscopy by Junghans et al. [41] who found that the walls became more permeable and allowed greater fluid transfer during recovery from myopia in the chicken.

\section{Muscarinic Control of Choroidal Thickness}

There is some evidence from work in chickens that inhibition of myopia development by atropine may not (only) be mediated by muscarinic mechanisms such as (1) atropine inhibits myopia even after cholinergic amacrine cells were ablated [42], (2) multiple muscarinic antagonists had little effect on myopia [43], and (3) receptor affinities did not correlate with myopia inhibition [44]. On the other hand, Meriney and Pilar [45] found in 1 day old chickens that "the choroidal coat is innervated by a dense network of cholinergic nerves that make en passant 
synapses with smooth muscle stimulation; of these nerve initiates, the red blood cell movement in the vessels of the choroidal coat, and this activation is blocked by muscarinic ACh receptor (AChR) antagonists." Also, Nickla et al. [46] provided evidence for a muscarinic control of choroidal thickness changes in chickens. In their study, choroidal thickness was measured by high frequency Ascan ultrasonography. In vivo, the muscarinic agonist oxotremorine triggered an increase in the axial eye growth together with choroidal thinning, while 2 other agonists (carbachol and arecaidine) only thinned the choroid after $24 \mathrm{~h}$. Choroidal thinning could also be elicited with the same agents in an eye cup preparation, again measured after $24 \mathrm{~h}$. In turn, muscarinic antagonists' atropine, pirenzepine, and oxyphenonium caused choroidal thickening in vivo, and the effects were already apparent after $3 \mathrm{~h}$. Also in our study, the peak of the choroidal response was between 2 and $3 \mathrm{~h}$.

While there is evidence that an increase in choroidal blood flow may trigger thickening of the choroid [33], it is not known whether atropine can increase choroidal blood flow in humans $[47,48]$. Figure $1 \mathrm{~b}$ shows that choroidal thickening occurs mainly on the scleral side of the choroid (which appears black in the OCT scans) and not in the choriocapillaris which is located adjacent to the pigment epithelium. Histological studies in chickens show that this area contains the lymphatic lacunae which can dynamically change their volume [14]. How atropine could act to expand lymphatic lacunae can only be speculated. There is also no published evidence of dopaminergic innervation to these lacunae. It is only known that dopaminergic agonists which inhibit myopia development also elicit a transient increase in choroidal thickness [49] and it is not known whether the latter change involves expansion of the lymphatic lacunae.

\section{Direct Effects of Atropine on Sclera}

Another unresolved question is whether atropine acts on molecular pathways that control both the thickness of the choroid and the growth of the sclera or whether choroidal thickening and scleral growth inhibition represent 2 separately controlled distinct mechanisms. There is some evidence for a scleral effect. Already in 1998, Lind et al. [50] found in scleral cell cultures that "chick scleral chondrocytes, synthesis of DNA, and glycosaminoglycans were inhibited by $\mathrm{mAChR}$ antagonists and, in vivo, the sclera may be a site of action for the mAChR antagonists previously used to influence myopia." Barathi and Beuerman [51] described an upregulation of mRNA levels for M1, M3, and M4 muscarinic acetylcholine recep- tors in the scleral tissue of mice which had been made myopic by wearing $-10 \mathrm{D}$ spectacle lenses. In cell culture, Cristaldi et al. [52] found that atropine-stimulated collagen I and fibronectin production in scleral fibroblasts, while it inhibited their production in choroidal fibroblasts. They concluded that atropine may act on both fundal layers, but differently, strengthening the scleral extracellular matrix but increasing permeability in the choroid. To better understand the effects of atropine on sclera, Hsiao et al. [53] applied $100 \mu \mathrm{M}$ atropine solution to primary human fibroblasts in culture for $24 \mathrm{~h}$ (with DMSO as control) and measured gene transcription levels. They found 168 genes downregulated and 206 upregulated. Canonical pathway analysis suggested scleral remodeling may be modulated by melatonin signaling pathways which supports a link between myopia and diurnal/circadian cycles. Such a link was frequently proposed (reviews $[54,55]$ ).

\section{Effects of Atropine on Dopamine Metabolism in}

Retina, Vitreous, and Choroid

Possible interactions between atropine and dopamine have been studied in the chicken model in the past by Schmid and Wildsoet [56]. These authors found that the suppressive effects of a dopamine agonist, apomorphine, and the muscarinic antagonist atropine on both deprivation myopia and negative lens-induced myopia were not additive, and proposed that "apomorphine and atropine act at different sites on a common control pathway" to inhibit myopia. They conclude that "combining dopaminergic and muscarinic agents is not a useful strategy." However, they did not study how atropine might act on dopamine production or release. It was striking that the time course of retinal dopamine release, induced by atropine injections [3], and the time course of choroidal thickening follows similar patterns. Both display a peak amplitude after about $2 \mathrm{~h}$. Normal diurnal cycles of retinal dopamine content and release [57] and choroidal thickness follow a similar pattern [58] and Nickla et al. [49] had shown that dopamine agonists can thicken the choroid. Furthermore, it was previously found that retinal dopamine temporarily increases in the vehicle-injected fellow eyes also [3], just like choroidal thickness, as found in the current study. Therefore, it is possible that there is a causal link.

While atropine increased the dopamine content in retina, it had the opposite effect in the choroid. Both choroidal dopamine and DOPAC levels were significantly lower in atropine-treated eyes. A possible explanation is that the expansion of the lymphatic lacunae may result in dilution 
of dopamine and its metabolites in the choroidal tissue. It was also striking that the regulation of choroidal thickness was linked in both eyes after unilateral atropine injection but no yoking was found in vitreal, retinal, and choroidal dopamine contents. Since we determined choroidal dopamine and metabolite concentrations relative to tissue wet weight, such a yoking effect should have shown up.

\section{Conclusions}

A key observation in our experiments in the chicken was that atropine exerts its effect only for a limited period of time, even at relatively high doses, sufficient to completely block myopia development. The time course was similar for dopamine content in the retina although this result does, of course, not prove that choroidal thickness changes are controlled by dopamine. We found that repeated atropine applications generated almost the same diurnal pattern of choroidal thickening with no signs of accumulation or desensitization over 4 days. Furthermore, we found that choroidal thickening in fellow eyes was also prominent, although more transient, likely due to the lower contralateral atropine concentrations. In general, the transient effects of atropine on the choroid may need to be kept in mind when application regimens are defined for atropine treatment against myopia.

\section{Acknowledgements}

We thank Sandra Bernhard-Kurz for excellent technical assistance.

\section{Statement of Ethics}

No studies were carried out involving humans. All experiments were conducted involving animals in accordance with the ARVO statement for the use of animals in ophthalmic and vision research and were approved by the official authorities at the University of Tuebingen (reference AK-01/19G).

\section{Conflict of Interest Statement}

The authors have no conflicts of interest to declare.

\section{Funding Sources}

We thank the German Research Council for the financial support (DFG Scha 518/15-1). We acknowledge the support by Open Access Publishing Funds by the University of Tuebingen.

\section{Author Contributions}

U.M. did the choroidal thickness measurements together with F.S., analyzed the HPLC data together with M.F. and wrote the manuscript. M.F. did the HPLC measurements, helped during statistical analyses and commented on the manuscript, and F.S. did experiments and wrote the manuscript. All authors analyzed and discussed the data.

\section{References}

1 Morgan IG, He M. An important step forward in myopia prevention: low-dose atropine. Ophthalmology. 2016 Feb;123(2):232-3.

2 Upadhyay A, Beuerman RW. Biological mechanisms of atropine control of myopia. Eye Contact Lens. 2020 May;46(3):129-35.

3 Mathis U, Feldkaemper M, Wang M, Schaeffel F. Studies on retinal mechanisms possibly related to myopia inhibition by atropine in the chicken. Graefes Arch Clin Exp Ophthalmol. 2020 Feb;258(2):319-33.

4 Feldkaemper M, Schaeffel F. An updated view on the role of dopamine in myopia. Exp Eye Res. 2013 Sep;114:106-19.

5 Zhou X, Pardue MT, Iuvone PM, Qu J. Dopamine signaling and myopia development: what are the key challenges. Prog Retin Eye Res. 2017 Nov;61:60-71.

6 Bitzer M, Kovacs B, Feldkaemper M, Schaeffel F. Effects of muscarinic antagonists on ZENK expression in the chicken retina. Exp Eye Res. 2006 Mar;82(3):379-88.
7 Ashby R, McCarthy CS, Maleszka R, Megaw P, Morgan IG. A muscarinic cholinergic antagonist and a dopamine agonist rapidly increase ZENK mRNA expression in the form-deprived chicken retina. Exp Eye Res. 2007 Jul;85(1):1522.

8 Torii H, Kurihara T, Seko Y, Negishi K, Ohnuma K, Inaba $\mathrm{T}$, et al. Violet light exposure can be a preventive strategy against myopia progression. EBioMedicine. 2017 Feb15;15:210-9.

9 Sander BP, Collins MJ, Read SA. Short-term effect of low-dose atropine and hyperopic defocus on choroidal thickness and axial length in young myopic adults. J Ophthalmol. 2019 Aug 21;2019:4782536.

10 Zhang Z, Zhou Y, Xie Z, Chen T, Gu Y, Lu S, et al. The effect of topical atropine on the choroidal thickness of healthy children. Sci Rep. 2016 Oct 7;6:34936.
11 Li W, Jiang R, Zhu Y, Zhou J, Cui C. Effect of $0.01 \%$ atropine eye drops on choroidal thickness in myopic children. J Fr Ophtalmol. Epub 2020 Aug 19;S0181-5512(20)30352-1.

12 Chiang ST, Phillips JR. Effect of atropine eye drops on choroidal thinning induced by hyperopic retinal defocus. J Ophthalmol. 2018 Jan 14; 2018:8528315.

13 Chiang ST, Turnbull PRK, Phillips JR. Additive effect of atropine eye drops and short-term retinal defocus on choroidal thickness in children with myopia. Sci Rep. 2020 Oct 27;10(1):18310.

14 Wallman J, Wildsoet C, Xu A, Gottlieb MD Nickla DL, Marran L, et al. Moving the retina: choroidal modulation of refractive state. Vision Res. 1995 Jan;35(1):37-50.

15 Liou HL, Brennan NA. Anatomically accurate, finite model eye for optical modeling. J Opt Soc Am A Opt Image Sci Vis. 1997 Aug; 14(8): 1684-95. 
16 Nickla DL, Totonelly K. Choroidal thickness predicts ocular growth in normal chicks but not in eyes with experimentally altered growth. Clin Exp Optom. 2015 Nov;98(6):564-70.

17 Nickla DL, Wildsoet C, Wallman J. Visual influences on diurnal rhythms in ocular length and choroidal thickness in chick eyes. Exp Eye Res. 1998 Feb;66(2):163-81.

18 Nickla DL, Wilken E, Lytle G, Yom S, Mertz J. Inhibiting the transient choroidal thickening response using the nitric oxide synthase inhibitor l-NAME prevents the ameliorative effects of visual experience on ocular growth in two different visual paradigms. Exp Eye Res. 2006 Aug; 83(2):456-64.

19 Jiang L, Garcia MB, Hammond D, Dahanayake D, Wildsoet CF. Strain-dependent differences in sensitivity to myopia-inducing stimuli in guinea pigs and role of choroid. Invest Ophthalmol Vis Sci. 2019 Mar 1;60(4):1226-33.

20 Troilo D, Nickla DL, Wildsoet CF. Choroidal thickness changes during altered eye growth and refractive state in a primate. Invest Ophthalmol Vis Sci. 2000 May;41(6):1249-58.

21 Hung LF, Wallman J, Smith EL 3rd. Vision-dependent changes in the choroidal thickness of macaque monkeys. Invest Ophthalmol Vis Sci. 2000;41(6):1259-69.

22 Wang D, Chun RK, Liu M, Lee RP, Sun Y, Zhang $\mathrm{T}$, et al. Optical defocus rapidly changes choroidal thickness in schoolchildren. PLoS One. 2016 Aug 18;11(8):e0161535.

23 Read SA, Alonso-Caneiro D, Vincent SJ, Collins MJ. Longitudinal changes in choroidal thickness and eye growth in childhood. Invest Ophthalmol Vis Sci. 2015 May;56(5):3103-12.

24 Jin P, Zou H, Xu X, Chang TC, Zhu J, Deng J, et al. Longitudinal changes in choroidal and retinal thicknesses in children with myopic shift. Retina. 2019 Jun;39(6):1091-9.

25 Prousali E, Haidich A-B, Fontalis A, Ziakas N, Brazitikos P, Mataftsi A. Efficacy and safety of interventions to control myopia progression in children: an overview of systematic reviews and meta-analyses. BMC Ophthalmol. 2019 May 9; 19(1):106.

26 Fontaine M, Gaucher D, Sauer A, Speeg-Schatz C. Choroidal thickness and ametropia in children: a longitudinal study. Eur J Ophthalmol. 2017 Nov 8;27(6):730-4.

27 Diether S, Schaeffel F, Lambrou GN, Fritsch C, Trendelenburg AU. Effects of intravitreally and intraperitoneally injected atropine on two types of experimental myopia in chicken. Exp Eye Res. 2007 Feb;84(2):266-74.

28 Ohngemach S, Hagel G, Schaeffel F. Concentrations of biogenic amines in fundal layers in chickens with normal visual experience, deprivation, and after reserpine application. Vis Neurosci. 1997 May-Jun;14(3):493-505.

29 Schaeffel F, Bartmann M, Hagel G, Zrenner E. Studies on the role of the retinal dopamine/ melatonin system in experimental refractive errors in chickens. Vision Res. 1995 May;35(9): 1247-64.
30 Li XX, Schaeffel F, Kohler K, Zrenner E. Dosedependent effects of 6-hydroxy dopamine on deprivation myopia, electroretinograms, and dopaminergic amacrine cells in chickens. Vis Neurosci. 1992 Nov;9(5):483-92.

31 Mathis U, Ziemssen F, Schaeffel F. Effects of a human VEGF antibody (Bevacizumab) on deprivation myopia and choroidal thickness in the chicken. Exp Eye Res. 2014 Oct;127:161-9.

32 Lan W, Feldkaemper M, Schaeffel F. Bright light induces choroidal thickening in chickens. Optom Vis Sci. 2013 Nov;90(11):1199-206.

33 Liu H, Schaeffel F, Trier K, Feldkaemper M. Effects of 7-methylxanthine on deprivation myopia and retinal dopamine release in chickens. Ophthalmic Res. 2020;63(3):347-57.

34 Wang M, Aleman AC, Schaeffel F. Probing the potency of artificial dynamic ON or OFF stimuli to inhibit myopia development. Invest Ophthalmol Vis Sci. 2019 Jun;60(7):2599-611.

35 Barathi VA, Beuerman RW, Schaeffel F. Effects of unilateral topical atropine on binocular pupil responses and eye growth in mice. Vision Res. 2009;49(3):383-7.

36 Wilson KT, Sivak JG, Callender MG. Induced refractive anomalies affect chick orbital bone structure. Exp Eye Res. 1997 May;64(5):67582.

37 Nickla DL, Wallman J. The multifunctional choroid. Prog Retin Eye Res. 2010 Mar;29(2): 144-68.

38 Fitzgerald ME, Wildsoet CF, Reiner A. Temporal relationship of choroidal blood flow and thickness changes during recovery from form deprivation myopia in chicks. Exp Eye Res. 2002 May;74(5):561-70.

39 Rada JA, Palmer L. Choroidal regulation of scleral glycosaminoglycan synthesis during recovery from induced myopia. Invest Ophthalmol Vis Sci. 2007 Jul;48(7):2957-66.

40 Pendrak K, Papastergiou GI, Lin T, Laties AM, Stone RA. Choroidal vascular permeability in visually regulated eye growth. Exp Eye Res. 2000 May;70(5):629-37.

41 Junghans BM, Crewther SG, Liang H, Crewther DP. A role for choroidal lymphatics during recovery from form deprivation myopia? Optom Vis Sci. 1999 Nov;76(11):796-803.

42 Fischer AJ, McKinnon LA, Nathanson NM, Stell WK. Identification and localization of muscarinic acetylcholine receptors in the ocular tissues of the chick. J Comp Neurol. 1998 Mar 16;392(3):273-84.

43 Luft WA, Ming Y, Stell WK. Variable effects of previously untested muscarinic receptor antagonists on experimental myopia. Invest Ophthalmol Vis Sci. 2003 Mar;44(3):1330-8.

44 Carr BJ, Mihara K, Ramachandran R, Saifeddine M, Nathanson NM, Stell WK, et al. Myopia-inhibiting concentrations of muscarinic receptor antagonists block activation of alpha2Aadrenoceptors in vitro. Invest Ophthalmol Vis Sci. 2018;59(7):2778-91.

45 Meriney SD, Pilar G. Cholinergic innervation of the smooth muscle cells in the choroid coat of the chick eye and its development. J Neurosci. 1987 Dec;7(12):3827-39.
46 Nickla DL, Zhu X, Wallman J. Effects of muscarinic agents on chick choroids in intact eyes and eyecups: evidence for a muscarinic mechanism in choroidal thinning. Ophthalmic Physiol Opt. 2013 May;33(3):245-56.

47 Fuchsjäger-Mayrl G, Malec M, Amoako-Mensah T, Kolodjaschna J, Schmetterer L. Changes in choroidal blood flow during light/dark transitions are not altered by atropine or propranolol in healthy subjects. Vision Res. 2003 Sep; 43(20):2185-90.

48 Polska E, Luksch A, Schering J, Frank B, Imhof A, Fuchsjäger-Mayrl G, et al. Propranolol and atropine do not alter choroidal blood flow regulation during isometric exercise in healthy humans. Microvasc Res. 2003 Jan;65(1):39-44.

49 Nickla DL, Totonelly K, Dhillon B. Dopaminergic agonists that result in ocular growth inhibition also elicit transient increases in choroidal thickness in chicks. Exp Eye Res. 2010 Nov; 91(5):715-20

50 Lind GJ, Chew SJ, Marzani D, Wallman J. Muscarinic acetylcholine receptor antagonists inhibit chick scleral chondrocytes. Invest Ophthalmol Vis Sci. 1998 Nov;39(12):2217-31.

51 Barathi VA, Beuerman RW. Molecular mechanisms of muscarinic receptors in mouse scleral fibroblasts: Prior to and after induction of experimental myopia with atropine treatment. Mol Vis. 2011 Mar 9;17:680-92.

52 Cristaldi M, Olivieri M, Pezzino S, Spampinato G, Lupo G, Anfuso CD, et al. Atropine differentially modulates ECM production by ocular fibroblasts, and its ocular surface toxicity is blunted by colostrum. Biomedicines. $2020 \mathrm{Apr}$ 5;8(4):78.

53 Hsiao YT, Chang WA, Kuo MT, Lo J, Lin HC, Yen MC, et al. Systematic analysis of transcriptomic profile of the effects of low dose atropine treatment on scleral fibroblasts using next-generation sequencing and bioinformatics. Int $\mathrm{J}$ Med Sci. 2019 Nov 9;16(12):1652-67.

54 Nickla DL. Ocular diurnal rhythms and eye growth regulation: where we are 50 years after Lauber. Exp Eye Res. 2013 Sep;114:25-34.

55 Stone RA, Wei W, Sarfare S, McGeehan B, Engelhart KC, Khurana TS, et al. Visual image quality impacts circadian rhythm-related gene expression in retina and in choroid: a potential mechanism for ametropias. Invest Ophthalmol Vis Sci. 2020 May 11;61(5):13.

56 Schmid KL, Wildsoet CF. Inhibitory effects of apomorphine and atropine and their combination on myopia in chicks. Optom Vis Sci. 2004 Feb;81(2):137-47.

57 Megaw PL, Boelen MG, Morgan IG, Boelen MK. Diurnal patterns of dopamine release in chicken retina. Neurochem Int. 2006 Jan;48(1): 17-23.

58 Nickla DL, Wildsoet CF, Troilo D. Endogenous rhythms in axial length and choroidal thickness in chicks: implications for ocular growth regulation. Invest Ophthalmol Vis Sci. $2001 \mathrm{Mar}$ 42(3):584-8. 